\title{
Jahresinhaltsverzeichnis 52. Jahrgang 2004
}

\section{AUFSÄTZE}

Ruth Ayaß

Konversationsanalytische Medienforschung ....

Werner Früh / Carsten Wünsch / TDU-Unterhaltungsindex. Ein Instrument zur em-

Pascal Klopp pirischen Ermittlung von Unterhaltungserleben . . 4/515

Elisabeth Klaus / Margreth Lünenborg Cultural Citizenship. Ein kommunikationswissenschaftliches Konzept zur Bestimmung kultureller Teilhabe in der Mediengesellschaft .......... 2/193

Steffen Kolb Verlässlichkeit von Inhaltsanalysedaten. Reliabilitätstest, Errechnen und Interpretieren von Reliabilitätskoeffizienten für mehr als zwei Codierer . . . 3/335

Jörg Matthes

Die Schema-Theorie in der Medienwirkungsforschung: Ein unscharfer Blick in die „Black Box“? . 4/545

Jörg Matthes / Matthias Kohring

Die empirische Erfassung von Medien-Frames . . 1/56

Michael Niehaus

Was ist Prominenz im Fernsehen? $4 / 613$

Manfred Rühl

Ist eine Allgemeine Kommunikationswissenschaft möglich? Eine Autopolemik ................ 2/173

Bertram Scheufele

Framing-Effekte auf dem Prüfstand. Eine theoretische, methodische und empirische Auseinandersetzung mit der Wirkungsperspektive des FramingAnsatzes .......................... 1/30

Ulla Wischermann

Der Kommunikationsraum Internet als Gendered Space .......................... 2/214

\section{BERICHTE}

Michel Clement

Erfolgsfaktoren von Spielfilmen im Kino. Eine Übersicht der empirischen betriebswirtschaftlichen Literatur .......................... 2/250

Michael Jäckel / Sabine Wollscheid

Medienzeitbudgets im Vergleich. Eine Gegenüberstellung der Langzeitstudie Massenkommunikation und der Zeitbudgeterhebung des Statistischen Bundesamtes

Matthias Kohring / Jörg Matthes

Revision und Validierung einer Skala zur Erfassung von Vertrauen in Journalismus . . . . . . . . . . . . . 3 3/377

Melanie Krause / Christoph Klimmt / Das Kommunikationswerkzeug als Lifestyle-AccesBeate Schneider tagsästhetik des Mobiltelefons bei Jugendlichen .. 3/432 
Christoph Kuhlmann / Jens Wolling

Mirko Marr

Henk Erik Meier

Michael Meyen

Thomas Schuster

Sabine Trepte

Christian Zabel

\section{LITERATUR}

\section{Besprechungen}

Klaus Beck

Volker Boehme-Neßler

Stefan Dahlem

Paula Diehl
Fernsehen als Nebenbeimedium. Befragungsdaten und Tagebuchdaten im Vergleich . . . . . . . . . 3/386

Wer hat Angst vor der Digitalen Spaltung? Zur Haltbarkeit des Bedrohungsszenarios ......... 1/76

Kommerzialisierung und Marktkonstitution. Zur politischen Konstruktion des Sportrechtemarktes . 4/583

Mediennutzer in der späten DDR. Eine Typologie auf der Basis biografischer Interviews . . . . . . . 1/95

Nachrichten und Börsenkurse. Preiseffekte ökonomischer und nicht-ökonomischer Veröffentlichungen in den Nachrichtenmedien . . . . . . . . . . 4/613

Soziale Identität und Medienwahl. Eine binationale Studie zum Einfluss von Gender-Identität und nationaler Identität auf die Selektion unterhaltender Medieninhalte ..................... 2/230

Zeitwettbewerb deutscher Free-TV-Anbieter ... 3/412

Nikolaus Schmitt-Walter: Online-Medien als funktionale Alternative? Über die Konkurrenz zwischen den Mediengattungen. München, 2004 . . . . . . 4 4/637

Holger Eggs: Vertrauen im Electronic Commerce. Herausforderungen und Lösungsansätze. Wiesbaden, 2001

Sabine Einwiller: Vertrauen durch Reputation im Elektronischen Handel. Wiesbaden, 2003

Heiner Fuhrmann: Vertrauen im Electronic Commerce. Rechtliche Gestaltungsmöglichkeiten unter besonderer Berücksichtigung verbindlicher Rechtsgeschäfte und des Datenschutzes. Baden-Baden,

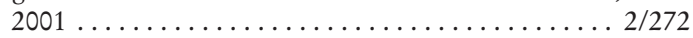

Christina Holtz-Bacha (Hrsg.): Die Massenmedien im Wahlkampf. Die Bundestagswahl 2002. Opladen, 2003 ... . . . . . . . . . . . . . . . . . . . . . . 4/639

Jochen Hoffmann: Inszenierung und Interpenetration. Das Zusammenspiel von Eliten aus Politik und Journalismus. Wiesbaden, 2003 .......... 3/455 
Patrick Donges

Johanna Dorer

Christa Dürscheid

Sandra Fröhlich

Rüdiger Funiok

Jörn Glasenapp

Alexander Görke

Udo Göttlich

Ulrike Gröttrup

Friederike von Gross / Uwe Sander

Thomas Hess

Knut Hickethier

Matthias Karmasin
Julian Weiss: Das Internet und die klassischen Medien. Konvergenz - Konkurrenz oder Komplementierung? Eine medienpolitische Betrachtung. Frankfurt a. M., 2003

Friederike Herrmann: Privatheit, Medien und Geschlecht. Bisexualität in Daily Talks. Opladen, 2002

Nicola Döring: Sozialpsychologie des Internet. Die Bedeutung des Internet für Kommunikationsprozesse, Identitäten, soziale Beziehungen und Gruppen. Göttingen u. a., 2003. . ............... 1/114

Vinzenz Wyss: Redaktionelles Qualitätsmanagement. Ziele, Normen, Ressourcen. Konstanz, 2002 ............................... 2/276

Gregor M. Jansen: Mensch und Medien. Entwurf einer Ethik der Medienrezeption. Frankfurt a. M., 2003 ............................. 4/640

Anton Holzer (Hrsg.): Mit der Kamera bewaffnet.

Krieg und Fotografie. Marburg: Jonas Verlag, 2003 2/278

Daniel Delhaes: Politik und Medien. Zur Interaktionsdynamik zweier sozialer Systeme. Wiesbaden,

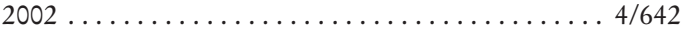

Matthias Karmasin / Carsten Winter (Hrsg.): Kulturwissenschaft als Kommunikationswissenschaft. Projekte, Probleme, Perspektiven. Wiesbaden, 2003 4/644

Irene Neverla / Elke Grittmann / Monika Pater (Hrsg.): Grundlagentexte zur Journalistik. Kons$\tan z, 2002 \ldots \ldots \ldots \ldots \ldots \ldots \ldots \ldots \ldots . \ldots \ldots 46 . \ldots \ldots$

Ingrid Paus-Haase / Claudia Lampert / Daniel Süss (Hrsg.): Medienpädagogik in der Kommunikationswissenschaft. Positionen, Perspektiven, Potenziale. Wiesbaden, 2002 ..................... 1/116

Thomas Schierl: Werbung im Fernsehen. Eine medienökonomische Untersuchung zur Effektivität und Effizienz werblicher TV-Kommunikation. Köln, 2003 ....................... 2/280

Hartmut Winkler: Diskursökonomie. Versuch über die innere Ökonomie der Medien. Frankfurt/M.,

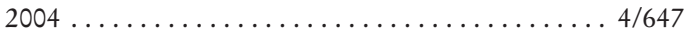

Thomas Breyer-Mayländer: Einführung in das Medienmanagement. Grundlagen, Strategie, Führung, Personal. München, 2004 .................. 3/457 
Matthias Knothe

Steffen Kolb

Hans-Dieter Kübler

Julian Kücklich

Christoph Kuhlmann

Claudia Lampert

Margreth Lünenborg

Margreth Lünenborg

Jürgen Maier

Michael Meyen

Burkard Michel
Martin Eifert: Konkretisierung des Programmauftrags des öffentlich-rechtlichen Rundfunks. Verfassungsrechtliche Verankerung, rechtliche Ausgestaltung und neue Herausforderungen der Selbstregulierung des öffentlich-rechtlichen Rundfunks. Baden-Baden, 2002 . . . . . . . . . . . . . . . . . . . . . .

Gerd G. Kopper / Paolo Mancini (Hrsg.): Kulturen des Journalismus und politische Systeme. Probleme internationaler Vergleichbarkeit des Journalismus in Europa - verbunden mit Fallstudien zu Großbritannien, Frankreich, Italien und Deutschland. Berlin, 2003

Burkhard Schäffer: Generationen - Medien - Bildung. Medienpraxiskulturen im Generationsvergleich. Opladen, $2003 \ldots \ldots \ldots \ldots \ldots \ldots \ldots \ldots$ 3/459

Klaus Walter: Grenzen spielerischen Erzählens. Spiel- und Erzählstrukturen in graphischen Adventure Games. Siegen, 2002 . . . . . . . . . . . . 1/122

Ulrich von Alemann / Stefan Marschall (Hrsg.): Parteien in der Mediendemokratie. Wiesbaden, 2002 . 1/124

Karsten Fritz / Stephan Sting / Ralf Vollbrecht (Hrsg.): Mediensozialisation. Pädagogische Perspektiven des Aufwachsens in Medienwelten. Opladen, $2003 \ldots \ldots \ldots \ldots \ldots \ldots \ldots \ldots \ldots \ldots . \ldots \ldots 4$. . . . . . . . . .

Johanna Dorer / Brigitte Geiger (Hrsg.): Feministische Kommunikations- und Medienwissenschaft. Ansätze, Befunde und Perspektiven der aktuellen Entwicklung. Wiesbaden, 2002

Julia Neissl (Hrsg.): der/die journalismus. Geschlechterperspektiven in den Medien. Innsbruck u. a., $2002 \ldots \ldots \ldots \ldots \ldots \ldots \ldots \ldots \ldots \ldots$. . . . . . . . . . . . .

Jörg-Uwe Nieland / Klaus Kamps (Hsrg.): Politikdarstellung und Unterhaltungskultur. Zum Wandel der politischen Kommunikation. Köln, 2003 . . 4/651

Markus Maurer: Politikverdrossenheit durch Medienberichte. Eine Paneluntersuchung. Konstanz,

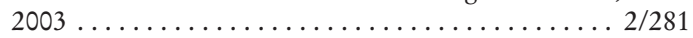

Ekkehardt Oehmichen / Christa-Maria Ridder (Hrsg.): Die MedienNutzerTypologie, Ein neuer Ansatz der Publikumsanalyse. Baden-Baden, 2003 1/128

Manuela Pietraß: Bild und Wirklichkeit. Zur Unterscheidung von Realität und Fiktion bei der Medienrezeption. Opladen, $2003 \ldots \ldots \ldots \ldots \ldots \ldots$. . . . . . . . . . 
Wolfgang Mühl-Benninghaus

Eggo Müller

Ute Nawratil

Christoph Neuberger

Stefan Ollig

Bernhard Pörksen

Thorsten Quandt

Dieter Roß

Helge Rossen-Stadtfeld

Manfred Rühl

Stephan Ruß-Mohl

Michael Schenk
Knut Hickethier: Einführung in die Medienwissenschaft. Stuttgart, 2003 ................. 3/464

Nathalie Ivanyi / Jo Reichertz: Liebe (wie) im Fernsehen. Eine wissenssoziologische Analyse. Opladen, 2002

Cornel Sandvoss: A Game of Two Halves. Football, Television and Globalization. London/New York, 2003

Michael Brüggemann: The Missing Link: Crossmediale Vernetzung von Print und Online. Fallstudien führender Print-Medien in Deutschland und den USA. München, 2002 . . . . . . . . . . . . . . . . . 4 4/654

Henning Never: Meinungsfreiheit, Wettbewerb und Marktversagen im Rundfunk. Eine ökonomische Kritik der verfassungsrechtlich geforderten positiven Rundfunkordnung. Baden-Baden, 2002 ..... 1/130

Stefan Weber (Hrsg.): Theorien der Medien. Von der Kulturkritik bis zum Konstruktivismus. Kons-

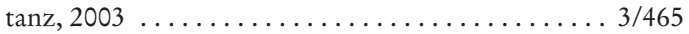

Kerstin Engels: Kommunikationsarbeit in OnlineMedien. Zur beruflichen Entwicklung kommunikativer Erwerbstätigkeiten - Eine explorative Studie aus institutionentheoretischer Sicht. Wiesbaden, 2003 . . . . . . . . . . . . . . . . . . . . . . . . 3/467

Werner Faulstich (Hrsg.): Die Kultur der 50er Jahre. München, 2002

Irmela Schneider / Peter M. Spangenberg (Hrsg.): Medienkultur der 50er Jahre. Wiesbaden, 2002 ... 2/284

Tarik Tabbara: Kommunikations- und Medienfreiheit in den USA. Zwischen demokratischen Aspirationen und kommerzieller Mobilisierung. BadenBaden, 2003 ........................ 2/286

Martin Löffelholz / Thorsten Quandt (Hrsg.): Die neue Kommunikationswissenschaft. Theorien, Themen und Berufsfelder im Internet-Zeitalter - eine Einführung. Wiesbaden, $2003 \ldots \ldots \ldots \ldots$. . . . . 1/132

Hans-Jürgen Bucher/Klaus-Dieter Altmeppen (Hrsg.): Qualität im Journalismus. Grundlagen Dimensionen - Praxismodelle. Wiesbaden, 2003 . . 3/469

Herbert Willems (Hrsg.): Die Gesellschaft der Werbung. Kontexte und Texte, Produktionen und Rezeptionen, Entwicklungen und Perspektiven. Wiesbaden, $2002 \ldots \ldots \ldots \ldots \ldots \ldots \ldots \ldots \ldots \ldots$. . . . . . . . . . . . 
Helmut Scherer

Roland Schröder

Roland Schröder

Margarete Schuler-Harms

Wolfgang Schweiger

Monika Suckfüll

Joachim Trebbe

Rainer Winter

Zeitschriftenlese

Literaturverzeichnis

\section{CHRONIK}

Christiane Matzen

English abstracts
Daniel Egloff: Digitale Demokratie: Mythos oder Realität? Auf den Spuren der demokratischen Aspekte des Internets und der Computerkultur. Wiesbaden, 2002 ..................... 4/659

Klaus-Dieter Altmeppen / Walter Hömberg (Hrsg.): Journalistenausbildung für eine veränderte Medienwelt. Wiesbaden, 2002 . . . . . . . . . . 1/133

Romy Fröhlich/Christina Holtz-Bacha (Hrsg.): Journalism Education in Europe and North America. An International Comparison. Cresskill, NJ,

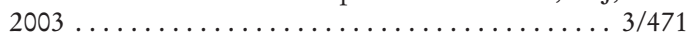

Michaela S. Tschon: Cross Ownership und publizistische Gewaltenteilung. Rechtstatsächliche Grundlagen und rechtliche Zulässigkeit der marktübergreifenden Eigentumskonzentration in den Medien. Zugleich ein Beitrag zur Dogmatik der Cross Ownership-Beschränkung unter besonderer Berücksichtigung des $\$ 26$ Abs. 2 Satz $2 \mathrm{RStV}$. Berlin,

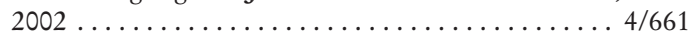

Jo Groebel / Gernot Gehrke (Hrsg.): Internet 2002: Deutschland und die digitale Welt. Internetnutzung und Medieneinschätzung in Deutschland und Nordrhein-Westfalen im internationalen Vergleich. Opladen, 2003 ...................... 4/663

Werner Früh: Unterhaltung durch das Fernsehen. Eine molare Theorie. Unter Mitarbeit von AnneKathrin Schulze und Carsten Wünsch. Konstanz, $2002 \ldots \ldots \ldots \ldots \ldots \ldots \ldots \ldots \ldots \ldots \ldots . \ldots \ldots .2 / 288$

Heinz Bonfadelli: Medieninhaltsforschung. Grundlagen, Methoden, Anwendungen. Konstanz, 2002 . 4/665

Ralph Weiß / Jo Groebel (Hrsg.): Privatheit im öffentlichen Raum. Medienhandeln zwischen Individualisierung und Entgrenzung. Opladen, 2002 . . 4/666

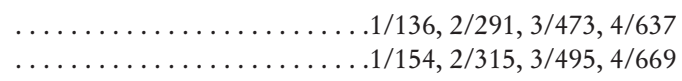

Chronik der Medienentwicklung in Deutschland $2003 \ldots \ldots \ldots \ldots \ldots \ldots \ldots \ldots \ldots \ldots \ldots \ldots . \ldots \ldots 4$ $.1 / 162,2 / 322,3 / 501,4 / 721$ 